ELECTRONIC RESEARCH ANNOUNCEMENTS OF THE AMERICAN MATHEMATICAL SOCIETY

Volume 3, Pages 38-44 (May 2, 1997)

S $1079-6762(97) 00021-8$

\title{
WEIGHTED POLYNOMIAL APPROXIMATION IN THE COMPLEX PLANE
}

\author{
IGOR E. PRITSKER AND RICHARD S. VARGA
}

(Communicated by Yitzhak Katznelson)

\begin{abstract}
Given a pair $(G, W)$ of an open bounded set $G$ in the complex plane and a weight function $W(z)$ which is analytic and different from zero in $G$, we consider the problem of the locally uniform approximation of any function $f(z)$, which is analytic in $G$, by weighted polynomials of the form $\left\{W^{n}(z) P_{n}(z)\right\}_{n=0}^{\infty}$, where $\operatorname{deg} P_{n} \leq n$. The main result of this paper is a necessary and sufficient condition for such an approximation to be valid. We also consider a number of applications of this result to various classical weights, which give explicit criteria for these weighted approximations.
\end{abstract}

\section{INTRODUCTION AND GENERAL RESULT}

In this paper, we will examine pairs of the form

$$
(G, W)
$$

where

(i) $G$ is an open bounded set, in the complex plane $\mathbb{C}$, which can be represented as a finite or countable union of disjoint simply connected domains, i.e., $G=\bigcup_{\ell=1}^{\sigma} G_{\ell}$ (where $1 \leq \sigma \leq \infty$ );

(ii) $W(z)$, the weight function, is analytic in $G$ with $W(z) \neq 0$ for any $z \in G$.

We say that the pair $(G, W)$ has the approximation property if,

$$
\left\{\begin{array}{l}
\text { for any } f(z) \text { which is analytic in } G \text { and for any compact subset } \\
E \text { of } G \text {, there exists a sequence of polynomials }\left\{P_{n}(z)\right\}_{n=0}^{\infty} \text {, with } \\
\operatorname{deg} P_{n} \leq n \text { for all } n \geq 0, \text { such that } \\
\qquad \lim _{n \rightarrow \infty}\left\|f-W^{n} P_{n}\right\|_{E}=0,
\end{array}\right.
$$

where all norms throughout this paper are the uniform (Chebyshev) norms on the indicated sets.

Given a pair $(G, W)$, as in (1.2), we state our main result, Theorem 1.1, which gives a characterization, in terms of potential theory, for the pair $(G, W)$ to have the approximation property. For notation, let $\mathcal{M}(E)$ be the space of all positive

Received by the editors October 15, 1996.

1991 Mathematics Subject Classification. Primary 30E10; Secondary 30C15, 31A15, 41 A30.

Key words and phrases. Weighted polynomials, locally uniform approximation, logarithmic potential, balayage. 
unit Borel measures on $\mathbb{C}$ which are supported on a compact set $E$, i.e., for any $\mu \in \mathcal{M}(E)$, we have $\mu(\mathbb{C})=1$ and $\operatorname{supp} \mu \subset E$. The logarithmic potential of a compactly supported measure $\mu$ is defined (cf. Tsuji $[13$, p. 53]) by

$$
U^{\mu}(z):=\int \log \frac{1}{|z-t|} d \mu(t) .
$$

Theorem 1.1. A pair $(G, W)$, as in (1.2), has the approximation property (1.3) if and only if there exist a measure $\mu(G, W) \in \mathcal{M}(\partial G)$ and a constant $F(G, W)$ such that

$$
U^{\mu(G, W)}(z)-\log |W(z)|=F(G, W), \quad \text { for any } z \in G .
$$

Remark 1.2. It is well known that any open set in the complex plane is a finite or countable union of disjoint domains, and this is more general than the assumption on the open set $G$ in (1.2)(i). However, we note that the approximation property (1.3) cannot hold, even in the classical case where $W(z) \equiv 1$ for all $z \in G$, if $G=\bigcup_{\ell=1}^{\sigma} G_{\ell}$, when some $G_{\ell}$ is multiply connected (cf. Walsh [14, p. 25]). In this sense, our initial assumptions on $G$ are quite general.

Remark 1.3. The condition that $W(z) \neq 0$ for all $z \in G$ cannot be dropped, for if $W\left(z_{0}\right)=0$ for some $z_{0} \in G_{\ell}$, where $G=\bigcup_{\ell=1}^{\sigma} G_{\ell}$, then the necessarily null sequence $\left\{W^{n}\left(z_{0}\right) P_{n}\left(z_{0}\right)\right\}_{n=0}^{\infty}$ trivially fails to converge to any $f(z)$, analytic in $G$, with $f\left(z_{0}\right) \neq 0$; whence, the approximation property fails. Even more decisive is the result that if $W\left(z_{0}\right)=0$ for some $z_{0} \in G_{\ell}$, then the sequence $\left\{W^{n}(z) P_{n}(z)\right\}_{n=0}^{\infty}$ can converge, locally uniformly in $G$, to $f(z)$, only if $f(z) \equiv 0$ in $G_{\ell}$. In this sense, the assumptions on $W(z)$ are also quite general.

Remark 1.4. In the case $W(z) \equiv 1$ of Theorem 1.1, the result that the approximation property (1.3) holds is a known classical result in complex approximation theory (cf. [14, p. 26]). This also follows from Theorem 1.1 because the measure $\mu(G, 1)$ exists by Theorems III.12 and III.14 of Tsuji [13], and is the classical equilibrium distribution measure (in the sense of logarithmic potential theory) for $\bar{G}$.

The topic of weighted approximation by $\left\{W^{n}(z) P_{n}(z)\right\}_{n=0}^{\infty}$, on the real line, has been extensively and thoroughly treated in the recent books of Saff and Totik [10] and Totik [12]. Here, we emphasize weighted approximation in the complex plane, which has received far less attention in the current approximation theory literature, with the exception of the recent papers by Borwein and Chen [1] and Pritsker and Varga [9].

We shall present in Section 2 a number of applications of Theorem 1.1 to special pairs $(G, W)$. The concluding Section 3 is devoted to further remarks, open problems and a discussion of possible generalizations.

\section{Applications}

Finding the measure $\mu(G, W)$ of Theorem 1.1 or verifying its existence is a nontrivial problem in general. Since $U^{\mu(G, W)}(z)$ is harmonic in $\mathbb{C} \backslash \operatorname{supp} \mu(G, W)$ and since it can be derived from (1.5) that if $\log |W(z)|$ is continuous on $\bar{G}$ and $G$ is a finite union of $G_{\ell}, \ell=1,2, \ldots, \ell_{0}$, then $U^{\mu(G, W)}(z)$ is equal to $\log |W(z)|+F(G, W)$ on supp $\mu(G, W)$, it follows that $U^{\mu(G, W)}(z)$ can be found as the solution of the corresponding Dirichlet problems. The measure $\mu(G, W)$ can be recovered from its 
potential, using the Fourier method described in Section IV.2 of Saff and Totik [10]. This method has already been used successfully by the authors in [9] to study the approximation of analytic functions by the weighted polynomials $\left\{e^{-n z} P_{n}(z)\right\}_{n=0}^{\infty}$, i.e., when $W(z):=e^{-z}$, and it is also used in the proof of Theorem 2.7.

In contrast to the above procedure, we next consider a different method, dealing with specific weight functions, which allows us to deduce "explicit" expressions for the measure $\mu(G, W)$ of Theorem 1.1, and to treat some important cases of pairs $(G, W)$. With $G$ as defined in (1.2)(i) for $\sigma$ finite, we denote the unbounded component of $\overline{\mathbb{C}} \backslash \bar{G}$ by $\Omega$. Let $\nu_{1}$ and $\nu_{2}$ be two unit positive Borel measures on $\mathbb{C}$ with compact supports satisfying

$$
\operatorname{supp} \nu_{1} \subset \overline{\mathbb{C}} \backslash G \text { and } \operatorname{supp} \nu_{2} \subset \overline{\mathbb{C}} \backslash G,
$$

such that

$$
\nu_{1}(\mathbb{C})=\nu_{2}(\mathbb{C})=1 .
$$

For real numbers $\alpha$ and $\beta$, assume that $W(z)$, satisfying

$$
\log |W(z)|=-\left(\alpha U^{\nu_{1}}(z)+\beta U^{\nu_{2}}(z)\right), \quad z \in G,
$$

is analytic in $G$. Then, as an application of Theorem 1.1, we state our next result:

Theorem 2.1. Given any pair of real numbers $\alpha$ and $\beta$, given an open bounded set $G=\bigcup_{\ell=1}^{\sigma} G_{\ell}$ as in (1.2)(i) with $\sigma$ finite, and given the weight function $W(z)$ of (2.3), then the pair $(G, W)$ has the approximation property (1.3) if and only if the measure

$$
\mu:=(1+\alpha+\beta) \omega(\infty, \cdot, \Omega)-\alpha \hat{\nu}_{1}-\beta \hat{\nu}_{2}
$$

is positive, where $\omega(\infty, \cdot, \Omega)$ is the harmonic measure at $\infty$ with respect to $\Omega$; here, $\hat{\nu}_{1}$ and $\hat{\nu}_{2}$ are, respectively, the balayages of $\nu_{1}$ and $\nu_{2}$ from $\overline{\mathbb{C}} \backslash \bar{G}$ to $\bar{G}$.

Furthermore, if $\mu$ of (2.4) is a positive measure, then (cf. Theorem 1.1)

$$
\mu(G, W)=\mu \text { and } \operatorname{supp} \mu(G, W) \subset \partial G .
$$

We point out that the harmonic measure $\omega(\infty, \cdot, \Omega)$ (cf. Nevanlinna [8] and Tsuji [13]) is the same as the equilibrium distribution measure for $\bar{G}$, in the sense of classical logarithmic potential theory [13]. For the notion of balayage of a measure, we refer the reader to Chapter IV of Landkof [5] or Section II.4 of Saff and Totik [10].

In the following series of subsections, we consider various classical weight functions and find their corresponding measures, associated with the weighted approximation problem in $G$ by Theorem 1.1 .

2.1. Incomplete polynomials and Laurent polynomials. With $\mathbb{N}_{0}$ and $\mathbb{N}$ denoting respectively the sets of nonnegative and positive integers, the incomplete polynomials of Lorentz [6] are a sequence of polynomials of the form

$$
\left\{z^{m(i)} P_{n(i)}(z)\right\}_{i=0}^{\infty}, \quad \operatorname{deg} P_{n(i)} \leq n(i),\left(m(i), n(i) \in \mathbb{N}_{0}\right),
$$

where it is assumed that $\lim _{i \rightarrow \infty} \frac{m(i)}{n(i)}=: \alpha$, where $\alpha>0$ is a real number. The question of the possibility of approximation by incomplete polynomials is closely connected to that of approximation by the weighted polynomials

$$
\left\{z^{\alpha n} P_{n}(z)\right\}_{n=0}^{\infty}, \quad \operatorname{deg} P_{n} \leq n .
$$


The question of approximation by the incomplete polynomials of (2.6) was completely settled by Saff and Varga [11], and by von Golitschek [3] on the interval [0,1] (see Lorentz, von Golitschek, and Makovoz [7], Totik [12], and Saff and Totik [10] for the associated history and later developments). We consider now the analogous problem in the complex plane. Since the weight $W(z):=z^{\alpha}$ in $(2.7)$ is multiplevalued in $\mathbb{C}$ if $\alpha \notin \mathbb{N}_{0}$, we then restrict ourselves to the slit domain $S_{1}:=\mathbb{C} \backslash(-\infty, 0]$ and the single-valued branch of $W(z)$ in $S_{1}$ satisfying $W(1)=1$.

For the related question of the approximation by the so-called Laurent polynomials

$$
\left\{\frac{P_{n(i)}(z)}{z^{m(i)}}\right\}_{i=0}^{\infty}, \quad \operatorname{deg} P_{n(i)} \leq n(i) \quad\left(m(i), n(i) \in \mathbb{N}_{0}\right)
$$

where $\lim _{i \rightarrow \infty} \frac{m(i)}{n(i)}:=\alpha, \alpha>0$, we are similarly led to the question of the approximation by the weighted polynomials

$$
\left\{z^{-\alpha n} P_{n}(z)\right\}_{n=0}^{\infty}, \quad \operatorname{deg} P_{n} \leq n,
$$

with the only difference being in the sign in the exponent of the weight function. Thus, we can give a unified treatment of both problems by considering weighted approximation by $\left\{W^{n}(z) P_{n}(z)\right\}_{n=0}^{\infty}, \operatorname{deg} P_{n} \leq n$, with

$$
W(z):=z^{\alpha}, \quad z \in S_{1}:=\mathbb{C} \backslash(-\infty, 0],
$$

where $\alpha$ is any fixed real number and where we choose, as before, the single-valued branch of $W(z)$ in $S_{1}$ satisfying $W(1)=1$.

Theorem 2.2. Given an open set $G$ as in (1.2)(i) with $\sigma$ finite, such that $\bar{G} \subset$ $S_{1}$, and given the weight function $W(z)$ of $(2.10)$, then the pair $(G, W)$ has the approximation property (1.3) if and only if

$$
\mu=(1+\alpha) \omega(\infty, \cdot, \Omega)-\alpha \omega(0, \cdot, \Omega)
$$

is a positive measure, where $\omega(\infty, \cdot, \Omega)$ and $\omega(0, \cdot, \Omega)$ are, respectively, the harmonic measures with respect to the unbounded component $\Omega$ of $\overline{\mathbb{C}} \backslash \bar{G}$, at $z=\infty$ and at $z=0$.

In some cases, when the geometric shape of $G$ is given explicitly, we can determine the explicit form of the measure of (2.11). This is especially easy to do for disks.

Corollary 2.3. Given the disk $D_{r}(a):=\{z \in \mathbb{C}:|z-a|<r\}$, where $a \in(0,+\infty)$ and $\bar{D}_{r}(a) \subset S_{1}=\mathbb{C} \backslash(-\infty, 0]$, i.e., $r<a$, and given the weight function of (2.10), then the pair $\left(D_{r}(a), W\right)$ has the approximation property (1.3) if and only if

$$
r \leq r_{\max }(a, \alpha)= \begin{cases}a, & \alpha \in[-1,0], \\ \frac{a}{|2 \alpha+1|}, & \alpha \in(-\infty,-1) \cup(0, \infty) .\end{cases}
$$

Furthermore, if (2.12) is satisfied, then the associated measure $\mu\left(D_{r}(a), z^{\alpha}\right)$ (see Theorem 1.1) is given by

$$
d \mu\left(D_{r}(a), z^{\alpha}\right)=\left(1+\alpha-\alpha \frac{a^{2}-r^{2}}{|z|^{2}}\right) \frac{d s}{2 \pi r},
$$

where $d s$ is the arclength measure on the circle $|z-a|=r$. 
2.2. Jacobi and Jacobi-type weights. We continue along the same lines by considering weighted approximation with Jacobi weights, i.e., we set

$$
W(z):=(1-z)^{\alpha}(1+z)^{\beta}, \quad z \in S_{2}:=\mathbb{C} \backslash\{(-\infty,-1] \cup[1, \infty)\},
$$

where $\alpha, \beta \in \mathbb{R}$ are any numbers, and where we choose the branch of weight function in $(2.14)$ such that $W(0)=1$.

An analogue of Theorem 2.2 in this case is the following result:

Theorem 2.4. Given an open set $G$ as in (1.2)(i) with $\sigma$ finite, such that $\bar{G} \subset$ $S_{2}$, and given the weight function $W(z)$ of $(2.14)$, then the pair $(G, W)$ has the approximation property (1.3) if and only if

$$
\mu=(1+\alpha+\beta) \omega(\infty, \cdot, \Omega)-\alpha \omega(1, \cdot, \Omega)-\beta \omega(-1, \cdot, \Omega)
$$

is a positive measure, where $\Omega$ is the unbounded component of $\overline{\mathbb{C}} \backslash \bar{G}$.

We next state a corollary of Theorem 2.4, which deals with the explicit formula for the radius of a largest disk $D_{r}(a)$, centered at $a \in(-1,1)$, for which $\left(D_{r}(a), W\right)$ has the approximation property.

Corollary 2.5. Given the disk $D_{r}(a):=\{z \in \mathbb{C}:|z-a|<r\}$, with $a \in(-1,1)$ and with $\bar{D}_{r}(a) \subset S_{2}$, and given the Jacobi weight function $W(z)$ of $(2.14)$, then the pair $\left(D_{r}(a), W\right)$ has the approximation property (1.3) if and only if

$$
1+\alpha+\beta-\alpha \frac{(1-a)^{2}-r^{2}}{|z-1|^{2}}-\beta \frac{(1+a)^{2}-r^{2}}{|z+1|^{2}} \geq 0 \text { on }|z-a|=r \text {. }
$$

In particular, if $\alpha \geq 0$ and $\beta \geq 0$, then the approximation property (1.3) holds if and only if

$$
\begin{aligned}
& r \leq r_{\max }(a, \alpha, \beta):= \\
& \frac{\sqrt{[\alpha-\beta+a(1+\alpha+\beta)]^{2}+\left(1-a^{2}\right)(1+2 \alpha+2 \beta)}-|\alpha-\beta+a(1+\alpha+\beta)|}{1+2 \alpha+2 \beta} .
\end{aligned}
$$

Furthermore, if (2.16) is valid, then

$$
\begin{aligned}
d \mu & \left(D_{r}(a),(1-z)^{\alpha}(1+z)^{\beta}\right) \\
\quad= & \left(1+\alpha+\beta-\alpha \frac{(1-a)^{2}-r^{2}}{|z-1|^{2}}-\beta \frac{(1+a)^{2}-r^{2}}{|z+1|^{2}}\right) \frac{d s}{2 \pi r}
\end{aligned}
$$

where $d s$ is the arclength measure on $|z-a|=r$.

Both weight functions, introduced in (2.10) and (2.14), are special cases of the following Jacobi-type weight function:

$$
W(z):=\prod_{i=1}^{p}\left(z-t_{i}\right)^{\alpha_{i}}
$$

where $\left\{\alpha_{i}\right\}_{i=1}^{p}$ are real numbers and $\left\{t_{i}\right\}_{i=1}^{p} \subset \mathbb{C}$ is a fixed set of distinct points. For a given open set $G$ (as in $(1.2)(\mathrm{i})$ with $\sigma$ finite) such that $t_{i} \notin \bar{G}, i=1, \ldots, p$, we assume that there exist $p$ cuts, connecting each $t_{i}$ with $\infty$. Then, we can define a single-valued branch of $W(z)$ in the $p$-slit complex plane which contains $\bar{G}$ in its interior. (It is impossible to specify those cuts in advance, as they necessarily depend on each preassigned open set $G$.) 
Theorem 2.6. The pair $(G, W)$ defined in the previous paragraph has the approximation property (1.3) if and only if

$$
\mu=\left(1+\sum_{i=1}^{p} \alpha_{i}\right) \omega(\infty, \cdot, \Omega)-\sum_{i=1}^{p} \alpha_{i} \omega\left(t_{i}, \cdot, \Omega\right)
$$

is a positive measure, where $\Omega$ is the unbounded component of $\overline{\mathbb{C}} \backslash \bar{G}$.

Furthermore, if $G=D_{r}(a):=\{z \in \mathbb{C}:|z-a|<r\}$ where $a \in \mathbb{C}$, then the pair $\left(D_{r}(a), W\right)$ has the approximation property $(1.3)$ if and only if

$$
1+\sum_{i=1}^{p} \alpha_{i}-\sum_{i=1}^{p} \alpha_{i} \frac{\left|t_{i}-a\right|^{2}-r^{2}}{\left|z-t_{i}\right|^{2}} \geq 0, \quad|z-a|=r .
$$

\subsection{Exponential weights. Let}

$$
W(z):=e^{-z^{m}}, \quad m \in \mathbb{N} .
$$

The special case $m=1$ of the weight function (2.22) was considered in [9]. To avoid technical complications, we shall study only the weighted approximation, with respect to the weight function $W(z)=e^{-z^{m}}$ in disks centered at the origin. Our next result generalizes Theorems 3.8 and 4.3 of [9].

Theorem 2.7. Given $D_{r}(0):=\{z \in \mathbb{C}:|z|<r\}$ and given the weight function $W(z)$ of $(2.22)$, then the pair $\left(D_{r}(0), W\right)$ has the approximation property (1.3) if and only if

$$
r \leq r_{\max }(m):=(2 m)^{-1 / m}, \quad m \in \mathbb{N} .
$$

Moreover, if (2.23) holds, then

$$
d \mu\left(D_{r}(0), e^{-z^{m}}\right)=\left(1-2 m r^{m} \cos m \theta\right) \frac{d \theta}{2 \pi},
$$

where $d \theta$ is the angular measure on $|z|=r$ and where $z=r e^{i \theta}$.

\section{FURTHER REMARKS AND OPEN PROBLEMS}

Theorem 1.1 gives a rather complete answer to the question on weighted approximation by $W^{n}(z) P_{n}(z)$ in open sets of the complex plane. It is then very natural to consider the uniform approximation by such weighted polynomials on compact sets, aiming at an analogue (generalization) of Mergelyan's theorem (see [14, p. $367]$ ). Let $E \subset \mathbb{C}$ be a compact set with connected complement $\overline{\mathbb{C}} \backslash E$. We denote the set of all functions, analytic interior to $E$ and continuous on $E$, by $A(E)$. Let $W \in A(E)$, with $W(z) \neq 0$ for any $z \in E$.

Problem. Give a necessary and sufficient condition for the pair $(E, W)$ to have the following approximation property:

For any $f \in A(E)$ there exist polynomials $\left\{P_{n}(z)\right\}_{n=0}^{\infty}$, with $\operatorname{deg} P_{n} \leq n$, such that

$$
\lim _{n \rightarrow \infty}\left\|f-W^{n} P_{n}\right\|_{E}=0 .
$$

Obviously, the classical uniform approximation by polynomials (Mergelyan's theorem) corresponds to $W(z) \equiv 1, z \in E$. We observe that (1.5) of Theorem 1.1, holding with $G=\operatorname{Int} E$, is a necessary condition for (3.1). Let us also remark that this problem is open even in the case when $E$ is a subset of the real line, such as 
an interval (see [10], [12] for background and general results, and Kuijlaars [4] for the recent progress in this area).

An even more general approach is to consider the approximation problem in (3.1) with polynomials replaced by rational functions. Certain results, concerning such weighted rational approximation were obtained by Borwein and Chen [1] in the complex plane, and by Borwein, Rakhmanov, and Saff [2] on the real line, for particular weights.

\section{REFERENCES}

[1] P. B. Borwein and W. Chen, Incomplete rational approximation in the complex plane, Constr. Approx. 11(1995), 85-106. MR 95k:41024

[2] P. B. Borwein, E. A. Rakhmanov and E. B. Saff, Rational approximation with varying weights I, Constr. Approx. 12(1996), 223-240. CMP 96:13

[3] M. von Golitschek, Approximation by incomplete polynomials, J. Approx. Theory 28(1980), 155-160. MR 81f:41005

[4] A. B. J. Kuijlaars, The role of the endpoint in weighted polynomial approximation with varying weights, Constr. Approx. 12(1996), 287-301. CMP 96:13

[5] N. S. Landkof, Foundations of modern potential theory, Springer-Verlag, Berlin, 1972. MR 50:2520

[6] G. G. Lorentz, Approximation by incomplete polynomials (problems and results), Padé and Rational Approximations: Theory and Applications (E. B. Saff and R. S. Varga, eds.), Academic Press, New York, 1977, pp. 289-302. MR 57:6956

[7] G. G. Lorentz, M. von Golitschek, and Y. Makovoz, Constructive approximation, SpringerVerlag, Berlin, 1996. CMP 96:13

[8] R. Nevanlinna, Analytic functions, Springer-Verlag, New York, 1970. MR 43:5003

[9] I. E. Pritsker and R. S. Varga, The Szegő curve, zero distribution and weighted approximation, to appear in Trans. Amer. Math. Soc. CMP 96:17

[10] E. B. Saff and V. Totik, Logarithmic potentials with external fields, Springer-Verlag, Heidelberg, 1996 (to appear).

[11] E. B. Saff and R. S. Varga, On incomplete polynomials, Numerische Methoden der Approximationstheorie, (eds. L. Collatz, G. Meinardus, and H. Werner), ISNM 42, Birkhäuser, Basel, 1978, 281-298. MR 80d:41008

[12] V. Totik, Weighted approximation with varying weights, Lecture Notes in Math., vol. 1569, Springer-Verlag, Heidelberg, 1994. MR 96f:41002

[13] M. Tsuji, Potential theory in modern function theory, Maruzen, Tokyo, 1959. MR 22:5712; MR 54:2990

[14] J. L. Walsh, Interpolation and approximation by rational functions in the complex domain, Colloquium Publications, vol. 20, Amer. Math. Soc., Providence, RI, 1965. MR 36:1672b

Institute for Computational Mathematics, Department of Mathematics and ComPuter Science, Kent State University, Kent, Ohio 44242-0001

E-mail address: pritsker@mcs.kent.edu

Institute for Computational Mathematics, Department of Mathematics and ComPuter Science, Kent State University, Kent, Ohio 44242-0001

E-mail address: varga@mcs.kent.edu 